\title{
Diets high in corn oil or extra-virgin olive oil differentially modify the gene expression profile of the mammary gland and influence experimental breast cancer susceptibility
}

\author{
Raquel Moral $^{1} \cdot$ Raquel Escrich $^{1} \cdot$ Montserrat Solanas $^{1} \cdot$ Elena Vela $^{1} \cdot$ \\ M. Carme Ruiz de Villa ${ }^{2} \cdot$ Eduard Escrich $^{1}$
}

Received: 7 October 2014 / Accepted: 9 June 2015 / Published online: 20 June 2015

(C) The Author(s) 2015. This article is published with open access at Springerlink.com

\begin{abstract}
Purpose Nutritional factors, especially dietary lipids, may have a role in the etiology of breast cancer. We aimed to analyze the effects of high-fat diets on the susceptibility of the mammary gland to experimental malignant transformation.

Methods Female Sprague-Dawley rats were fed a low-fat, high-corn-oil, or high-extra-virgin olive oil (EVOO) diet from weaning or from induction. Animals were induced with 7,12-dimethylbenz[a]anthracene at 53 days and euthanized at 36, 51, 100 and 246 days. Gene expression profiles of mammary glands were determined by microarrays. Further molecular analyses were performed by real-time PCR, TUNEL and immunohistochemistry. Carcinogenesis parameters were determined at 105 and 246 days.

Results High-corn-oil diet increased body weight and mass when administered from weaning. The EVOO diet did not modify these parameters and increased the hepatic expression of UCP2, suggesting a decrease in intake/ expenditure balance. Both diets differentially modified the gene expression profile of the mammary gland, especially
\end{abstract}

Raquel Moral and Raquel Escrich have been contributed equally to this work.

Electronic supplementary material The online version of this article (doi:10.1007/s00394-015-0958-2) contains supplementary material, which is available to authorized users.

Eduard Escrich

Eduard.Escrich@uab.cat

1 Physiology Unit, Department of Cell Biology, Physiology and Immunology, Faculty of Medicine, Universitat Autònoma de Barcelona, Bellaterra, 08193 Barcelona, Spain

2 Department of Statistics, Universitat de Barcelona, 08028 Barcelona, Spain after short dietary intervention. Corn oil down-regulated the expression of genes related to immune system and apoptosis, whereas EVOO modified the expression of metabolism genes. Further analysis suggested an increase in proliferation and lower apoptosis in the mammary glands by effect of the high-corn-oil diet, which may be one of the mechanisms of its clear stimulating effect on carcinogenesis.

Conclusions The high-corn-oil diet strongly stimulates mammary tumorigenesis in association with modifications in the expression profile and an increased proliferation/ apoptosis balance of the mammary gland.

Keywords Extra-virgin olive oil · Corn oil · Dietary lipids · Mammary gland · Experimental tumors · Gene expression profile

\section{Introduction}

Breast cancer is a leading cause of mortality in women worldwide [1]. Although the etiology of this neoplasia is clearly multifactorial, there is striking evidence of the influence of environmental factors, diet being one of the most important [2]. Human and especially experimental data have shown the effects of dietary lipids on breast cancer risk [2-4]. Epidemiological studies have reported some inconsistent data, likely because of the difficulties in precisely estimating fat intake [5]. However, new approaches from the European Prospective Investigation into Cancer and Nutrition (EPIC) have found significant positive associations of saturated and total fat intake with breast cancer risk $[6,7]$. On the other hand, animal models have provided a wealth of evidence for the relationship between dietary lipids and breast cancer. Thus, $\mathrm{n}-6$ polyunsaturated fatty acids (PUFA), particularly linoleic acid (18:2n-6), 
have shown a strong stimulating effect on breast, colorectal and prostate cancers. Saturated fat, mainly from animal origin, has also a stimulating influence on tumorigenesis. On the contrary, n-3 PUFA, conjugated linoleic acid and $\gamma$-linolenic acid, have demonstrated an inhibitory influence on carcinogenesis. The effects of monounsaturated fatty acids (MUFA) are still not well elucidated, although several studies reported a potential protective effect on experimental breast cancer $[3,4]$. In this sense, there is also epidemiological evidence that Mediterranean diet (characterized by the consumption of olive oil as the principal source of fat) has a protective effect on the risk of developing several types of tumors, including breast cancer [8, 9]. Lifestyle patterns including diet, physical activity and body weight have been associated with metabolic dysregulation, which may play a role in the association between lifestyle and the development of breast cancer. Actually, Mediterranean diet has also been inversely associated with body weight, adiposity and metabolic syndrome, in addition to other chronic diseases [10,11].

The mammary gland, unlike other organs, is not fully developed after birth. Through postnatal life, the female breast tissue undergoes several key developmental stages, being one of the most important puberty. There is extensive evidence that nutritional status and body size may contribute to maturation trends and breast cancer. Western countries lifestyle, characterized by high consumption of fat, has been associated with obesity and earlier pubertal maturation, known factors of breast cancer risk $[2,12]$. The effects of environmental factors, including nutritional ones, are dependent on the developmental stage when exposition occurs. In this regard, the influence of high-fat diets on experimental breast cancer risk may be different if dietetic intervention occurs in utero, before puberty or after sexual maturation $[13,14]$. Thus, high-fat diets may affect mammary development and thus the window where the gland is most susceptible to malignant transformation. Furthermore, in experimental models, dietary lipids may differentially influence the initiation (if exposure begins before carcinogen induction) or the promotion (if exposure begins after induction) of tumorigenesis [3].

The different effects that diets have on breast cancer susceptibility are probably driven by multiple and complex molecular mechanisms. Although such mechanisms are not fully understood, several have been suggested, including alterations of hormonal status, modifications of the structure and function of membranes, disruption of cell-signaling pathways and modulation of gene expression [4]. It is well known that dietary long-chain fatty acids modulate the transcription of genes involved in lipid metabolism. Although fewer data are published regarding the effects of lipids in cancer-related genes, there is a growing body of evidence about their role in the modulation of genes with a role in proliferation, differentiation and apoptosis [15]. Hence, the aim of this work is to analyze the influence of different high-fat diets, i.e., rich in corn oil or in extra-virgin olive oil (EVOO), administered in different periods (after weaning onwards or after induction onwards) on breast cancer susceptibility and to get insight into the underlying molecular mechanisms. For this extent, developmental changes have been monitored, including growth, body mass indexes and hepatic expression of metabolism genes at different ages. Gene expression profile of breast tissues has been determined at different developmental stages, including peri-puberty and adulthood. Experimental tumors have been induced with DMBA at 53 days of age, and clinical manifestation of carcinogenesis has been analyzed at different ages.

\section{Materials and methods}

\section{Diets}

Three semisynthetic diets were designed: a low-fat diet (at $3.71 \mathrm{kcal} / \mathrm{g}$, containing $7.3 \%$ calories under the form of fat) and two high-fat diets (at $4.56 \mathrm{kcal} / \mathrm{g}$, containing $39.5 \%$ calories in the form of fat). The control low-fat diet contained $3 \%$ corn oil (w/w), the high-corn-oil diet contained $20 \%$ of this same oil, while the high olive oil diet contained $3 \%$ corn oil $+17 \%$ extra-virgin olive oil. Carbohydrates in the form of dextrose were $67.9 \%-\mathrm{w} / \mathrm{w}-(73 \%$ calories) in the low-fat diet and $45.9 \%$-w/w- $(40.3 \%$ calories) in both high-fat diets. They also contained $20 \%$ of calories from proteins (18 $\mathrm{g}$ casein/100 $\mathrm{g}$ low-fat diet; $23 \mathrm{~g}$ casein $/ 100 \mathrm{~g}$ high-fat diet) and $5 \%$ - w/w-cellulose, $5.9 \%$ - w/w-salt mixture and $0.24 \%-\mathrm{w} / \mathrm{w}-$ vitamin mixture (Supplementary Table 1). In order to maintain the normal lipidic metabolism, they were supplemented with methionine $(0.51 \%-\mathrm{w} / \mathrm{w}-$ in the low-fat and $0.66 \%$ in the two high-fat diets), choline $(1800 \mathrm{mg} / \mathrm{kg}$ diet $)$ and folic acid $(5 \mathrm{mg} / \mathrm{kg}$ diet). Diets were prepared weekly and stored under nitrogen in the dark at $4{ }^{\circ} \mathrm{C}$. The definition, preparation and suitability of the diets have been previously described [16-19].

\section{Animals and experimental design}

All animals received humane care under an institutionally approved experimental animal protocol, following the legislation applicable in this country. Female Sprague-Dawley Crl:SD rats $(N=167$; Charles River Lab, L'Arbresle Cedex, France) were distributed depending on the type and timing of dietary intervention. Thus, from weaning onwards, control animals were fed the low-fat diet (group LF, $N=87$ ), while the high-fat groups animals were fed 


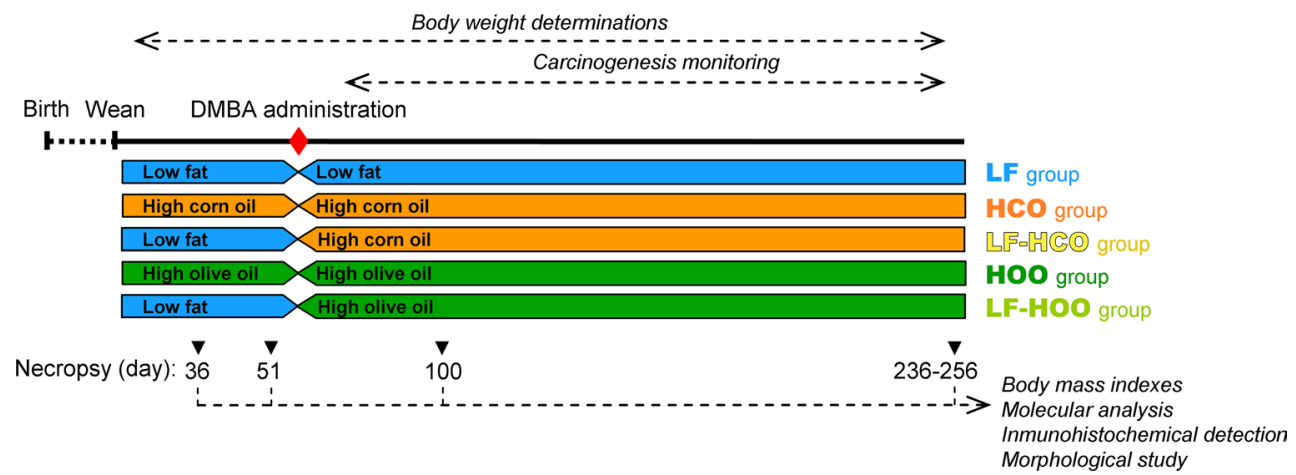

Fig. 1 Experimental design. Female Sprague-Dawley rats were fed the low-fat control diet (LF), the high-corn-oil diet from weaning (HCO) or from induction (LF-HCO), and the high-extra-virgin olive oil diet from weaning (HOO) or from induction (LF-HOO). Animals were induced with $5 \mathrm{mg}$ of dimethylbenz $[\alpha]$ anthracene (DMBA) at

the high-corn-oil diet (group HCO, $N=37$ ) or the highextra-virgin olive oil diet (group $\mathrm{HOO}, N=37$ ) and water ad libitum. At 53 days of age, mammary cancer was induced by oral gavage with one single dose of $5 \mathrm{mg}$ of DMBA (Sigma-Aldrich Inc, St Louis, MO, USA) dissolved in corn oil. To study the promotion of the carcinogenesis, after DMBA treatment, 50 rats from the LF group were changed to high-fat diets (groups LF-HCO, and LF-HOO, $N=25$ each) (Fig. 1).

Animals were examined and weighed weekly throughout the study. At days of killing, nose-to-anus length was also measured and the following body mass indexes were determined: BMI (body mass index, $\mathrm{g} / \mathrm{cm}^{2}$ ) and Lee index $(10,000 \times \mathrm{g} / 3 / \mathrm{cm})$ [20]. From day 74 onwards, all rats were monitored for mammary tumor appearance weekly. Carcinogenesis was evaluated at 105 days (52 days after DMBA induction) and at 236-256 days (median day 246-day 193 post-induction, end of the assay). The following carcinogenesis parameters were calculated: tumor incidence (percentage of tumor-bearing animals), tumor yield (total number of mammary tumors) and total tumor volume $\left(V=4 / 3 \pi\left[d_{1} / 2\right] \times\left[d_{2} / 2\right]^{2}\right.$, where $d_{1}$ and $d_{2}$ are the two diameters of the tumor $\left(d_{1}>d_{2}\right)$, and at killing with the three diameters $V=4 / 3 \pi$ $\left.\left[d_{1} / 2\right] \times\left[d_{2} / 2\right] \times\left[d_{3} / 2\right]\right)$.

Rats were euthanized by decapitation at day $36(N=6 /$ experimental condition: control, HCO, HOO), $51(N=6 /$ experimental condition: control, HCO, HOO), 100 ( $N=5 /$ group $)$ and the end of the assay $(N=20 /$ group $)$. Left abdominal mammary glands and liver were collected and flash-frozen for molecular analyses. Right mammary glands were fixed in buffered formalin for morphological and histological analyses. At the end of the assay, tumors were excised and a portion fixed in $4 \%$ formalin
53 days of age. Rats were euthanized at day $36(N=6$ /experimental condition: LF, HCO, HOO), day $51(N=6 /$ experimental condition: LF, HCO, HOO $)$, day $100(N=5 /$ group $)$ and at the end of the assay (236-256, median day $246 ; N=20 /$ group)

for histopathological diagnosis [21]. Only data from confirmed mammary adenocarcinomas have been included in this study.

\section{RNA isolation and gene expression analysis by real-time PCR}

Total RNA from liver and left abdominal mammary gland was extracted using the Tissue RNeasy Extraction Kit (QiaGen, Hilden, Germany). RNA was quantified spectrophotometrically with Nanodrop 1000 (ThermoFisher Scientific Inc, Waltham, MA, USA), and integrity was determined using a 2100 Bioanalyser (Agilent Technologies, Santa Clara, CA, USA). The study of expression of specific genes was performed by real-time PCR in the iCycler MyiQ RealTime PCR detection system (Bio-Rad Laboratories, Hercules, CA, USA). Analyses of mRNA levels were performed using the TaqMan methodology (Applied Biosystems Inc, Foster City, CA, USA). Two micrograms of total RNA was reverse-transcribed using the High Capacity cDNA Reverse Transcription Kit (Applied Biosystems). Reactions were prepared with the TaqMan Universal PCR Master Mix and the suitable TaqMan assay. For the study of metabolism genes, the following assays were used: Rn00566193_m1 $(\mathrm{PPAR} \alpha), \quad \mathrm{Rn} 00580702 \_\mathrm{m} 1$ (CPT1a), Rn00664587_m1 (L-FABP), Rn01754856_m1 (UCP2). For validation of arrays (see below), the following assays were used (in alphabetical order of the target gene): Rn00573474_m1 (Acaca), Rn00566411_m1 (Acly), Rn00595250_m1 (Adipoq), Rn01507624_m1 (Cd19), Rn00565469_m1 (Cd28), Rn00565890_m1 (Cd3d), Rn00560963_s1 (Cebpa), Rn01524626_m1 (Csn2- $\beta$ casein), Rn01511686_g1 (Hsp90ab1), Rn00574380_m1 (Insig1), Rn00565158_m1 (Lep), Rn00575662_m1 (Pgr), Rn00440945_m1 (PPAR $\gamma$ ), 
Rn00821474_g1 (S100a6) Rn00565937_m1 (Tgf $\beta 3)$, Rn00710369_m1 (Tp53inp1). Twenty nanograms of cDNA was amplified during 40 cycles of $15 \mathrm{~s}$ at $95{ }^{\circ} \mathrm{C}$ and $60 \mathrm{~s}$ at $60{ }^{\circ} \mathrm{C}$. Gene expression was normalized using Hprt (Rn01527840_m1) as a control transcript.

\section{Whole-genome gene expression profiling}

After quality control of total RNA using 2100 Bioanalyser (Agilent Technologies), only samples with integrity numbers $\geq 8$ were used for these analyses. For the determination of the effects of high-fat diets on the gene expression profile of the mammary gland at different ages, we used GeneChip $^{\circledR}$ Rat Exon 1.0 ST Array (Affymetrix, Santa Clara, CA, USA). Three mammary glands of each group at each age tested were chosen. For each sample, $3 \mu \mathrm{g}$ of total RNA was labeled, hybridized to chips and scanned, in the Microarrays Service from Vall d'Hebron Research Institute (VHIR).

\section{Microarray data analysis}

The scanned images were processed with Microarray Analysis Suite 5.0 (Affymetrix). Raw expression values were preprocessed using the RMA method [22] for background correction, normalization and summarization of probe values. The data obtained were then non-specific filtered to remove genes with low signal and low variability. The statistical analysis of data was performed using the free statistical language $\mathrm{R}$ (www.bioconductor.org), following the methods described by Gentleman et al. [23]. The selection of differentially expressed genes between conditions was based on a linear model analysis with empirical Bayes moderation of the variance estimates [24]. This method approximation is of special interest in microarray data analysis where sample sizes are small. $p$ values were adjusted to obtain strong control over the false discovery rate using the Benjamini and Hochberg method [25]. Microarray data have been deposited at ArrayExpress under accession number E-MTAB-3530.

Genes selected as differentially expressed (up- or down-regulated) were classified by their biological processes according to Gene Ontology (GO) database. Determination of biological significance was based on an overrepresentation analysis which establishes whether the differentially expressed genes appeared to be concentrated in GO categories corresponding to specific biological processes. This kind of enrichment tests was also performed using FatiGO from Babelomics tool. Fisher's exact test for $2 \times 2$ contingency tables and multiple test correction are applied to find significant overrepresentation of GO terms.

\section{Morphological analysis of the mammary gland}

Dissected right abdominal glands were fixed in $10 \%$ neutral-buffered formalin for $48 \mathrm{~h}$, defatted in acetone, rehydrated and stained in alum carmine. Tissues were dehydrated in graded alcohol, cleared in HistoChoice (Sigma-Aldrich), trimmed of excess of connective tissue under a stereomicroscope and coverslipped with mounting media. Microscopic epithelial proliferative structures were evaluated under a Nikon Eclipse E800 light microscope and the image software ACT-1 for DXM 1200F version 2.51 (Nikon Instruments Europe B.V., Amsterdam, Netherlands). Hyperplastic and neoplastic lesions were identified: hyperplasias, intra-ductal proliferations (IDP) and tumors.

\section{Apoptosis detection}

Tissues were fixed in $4 \%$ phosphate-buffered formalin, paraffin-embedded and cut in 5- $\mu \mathrm{m}$ sections. Apoptosis was assessed through terminal deoxynucleotidyl transferase dUTP nick end labeling (TUNEL) assay using the ApopTag Plus Peroxidase In Situ Detection Kit (Chemicon, Concord Road, MA, USA) according to the manufacturer protocol. Epithelial structures were identified in the mammary glands using the criteria previously established [26] (terminal end buds-TEB, ducts and lobules), and apoptotic cells were visualized under an Axiostar Plus microscope (Carl Zeiss Microscopy, Jena, Germany). To minimize variation, all samples were examined and photographed with the same microscope settings.

\section{Immunohistochemistry}

Infiltration of $\mathrm{T}$ cells in the different epithelial structures of the mammary gland was visualized by immunohistochemical detection of CD3-positive cells. Formalin-fixed paraffin-embedded tissue sections were incubated with a specific antibody against CD3 antigen (ab 16669, Abcam, Cambridge, UK) and a biotinylated anti-rabbit secondary IgG antibody (BA-1000, Vector Labs, Burlingame, CA, USA). Immunoreactivity was detected by incubation with chromogen diaminobenzidine (DAB) for 6 min. Tissue sections were counterstained with hematoxylin, dehydrated through graded ethanol and xylene, and cover-slipped. CD3-positive cells were visualized, and images were captured with an Axiostar Plus microscope (Carl Zeiss Microscopy).

\section{Other statistical analysis}

Body weight throughout the assay was analyzed using nonlinear mixed models. We used parametric or nonparametric statistics depending on the distribution of each variable 


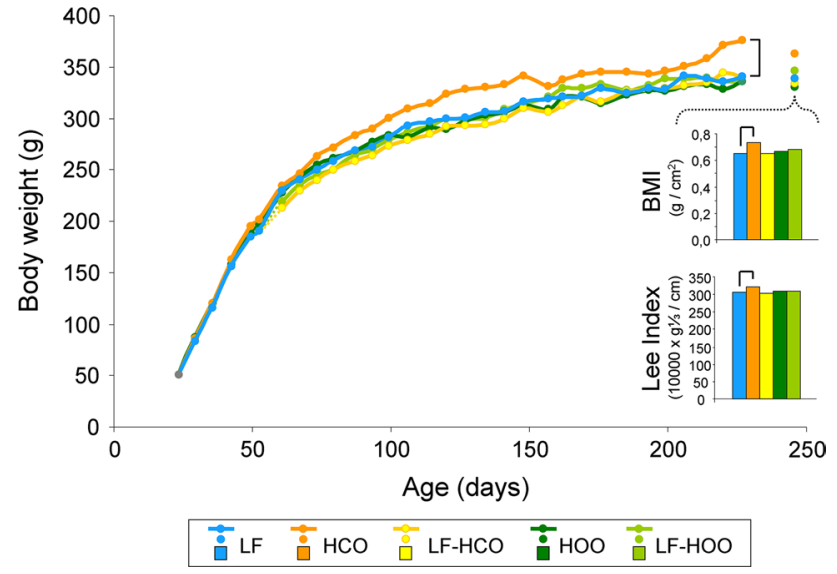

Fig. 2 Body-weight evolution and body mass indexes (BMI and Lee index) at the end of the assay (236-256 days, median 246 days). Data represent medians of the groups. For body-weight evolution, number of determinations was as follows: high-fat groups: $N=37$ (from 24 to 35 days), 31 (from 36 to 50 days), 25 (from 51 to 99 days) and 20 (from 100 onwards); control: $N=87$ (from 24 to 35 days), 81 (from 36 to 50 days), 75 (from 51 to 53 days), 25 (from 54 to 99 days) and 20 (from 100 onwards). Body mass indexes, $N=20 /$ group. Lines connecting groups indicate differences statistically significant $(p<0.05)$, nonlinear mixed models (body weight) and Mann-Whitney's $U$ test (body mass indexes)

studied, determined by Kolmogorov-Smirnov test, and the equality of variances among groups, determined by Levene's test. Parametric quantitative data were analyzed with ANOVA followed by Tukey's test. Analysis of nonparametric quantitative data was performed with Friedman and Mann-Whitney's $U$ tests. Since results along time corresponded to tissues from non-induced (36 and 51 days) and DMBA-induced (100 and 246 days) animals, and most of the data did not follow a normal distribution, in order to homogenize results, nonparametric statistic is represented. Qualitative data were analyzed with Pearson's Chi-squared test. Differences were considered significant when $p<0.05$.

\section{Results}

\section{Effects of high-fat diets on body weights and metabolism}

Animals fed the high-corn-oil diet from weaning (group HCO, Fig. 1) increased body weight (Fig. 2). In contrast, administration of the high EVOO throughout life (group HOO) did not modify body weight. These high-fat diets, administered after puberty (from induction onwards, i.e., groups LF-HCO and LF-HOO), had no effect on this parameter.

There were no differences in the body mass indexes at 36,51 and 100 days by effect of the high-fat diets (results
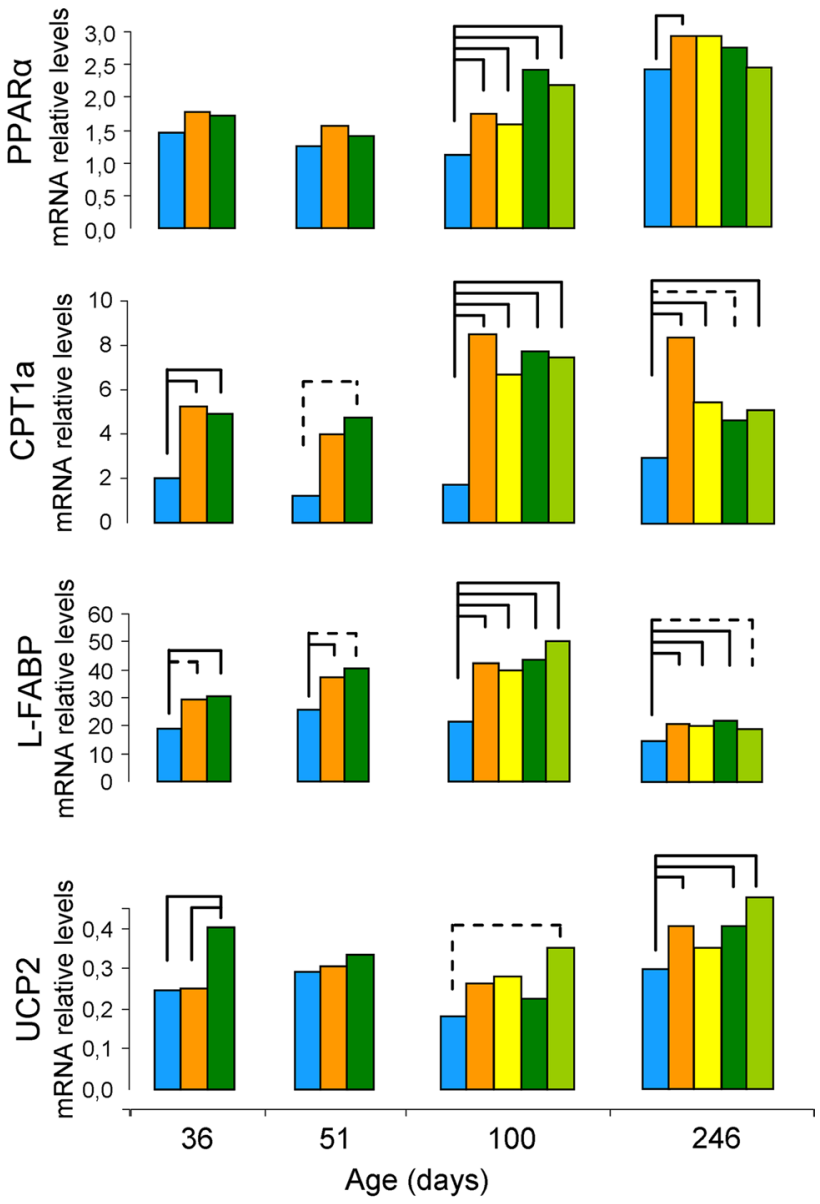

$\square$ LF $\square$ HCO $\square$ LF-HCO $\square$ HOO $\square$ LF-HOO

Fig. 3 Relative hepatic mRNA expression of genes with a role in lipid transport, metabolism and energy balance. Data shown represent medians of the groups ( 36 and 51 days $N=6$ /group; 100 days $N=5$ / group, 246 days $N=20 /$ group). Full lines connecting groups indicate differences statistically significant $(p<0.05)$, and dotted lines indicate differences close to significance $(p<0.1)$, Mann-Whitney's $U$ test

not shown). At the end of the assay, body mass index (BMI) and Lee index were increased in HCO group in relation to control LF group (Fig. 2).

High-fat diets modulated the hepatic expression of genes with a role in lipid metabolism and transport. Effects on peroxisome proliferator-activated receptor alpha (PPAR $\alpha)$ expression were only evident at 100 days, when all highfat diet groups increased the mRNA levels of this gene. All high-fat diet groups had also increased expression of carnitine palmitoyltransferase 1a (CPT1a) and liver-type fatty-acid-binding protein (L-FABP) compared to control group at all ages, most of these differences being statistically significant. Uncoupling protein 2 (UCP2) was increased specially by effect of the high EVOO diet, mainly around puberty (36 days; Fig. 3). 
Fig. 4 Number of sequences differentially expressed (Benjamini and Hochberg false discovery rate method) in mammary glands of animals fed the highfat diets $(N=3$ gene expression profiles/group/age). For each age, Venn diagrams depict overlapping genes within high-fat diet groups being significantly different from low-fat control group. Black italic numbers represent down-regulated genes, and red numbers represent upregulated genes

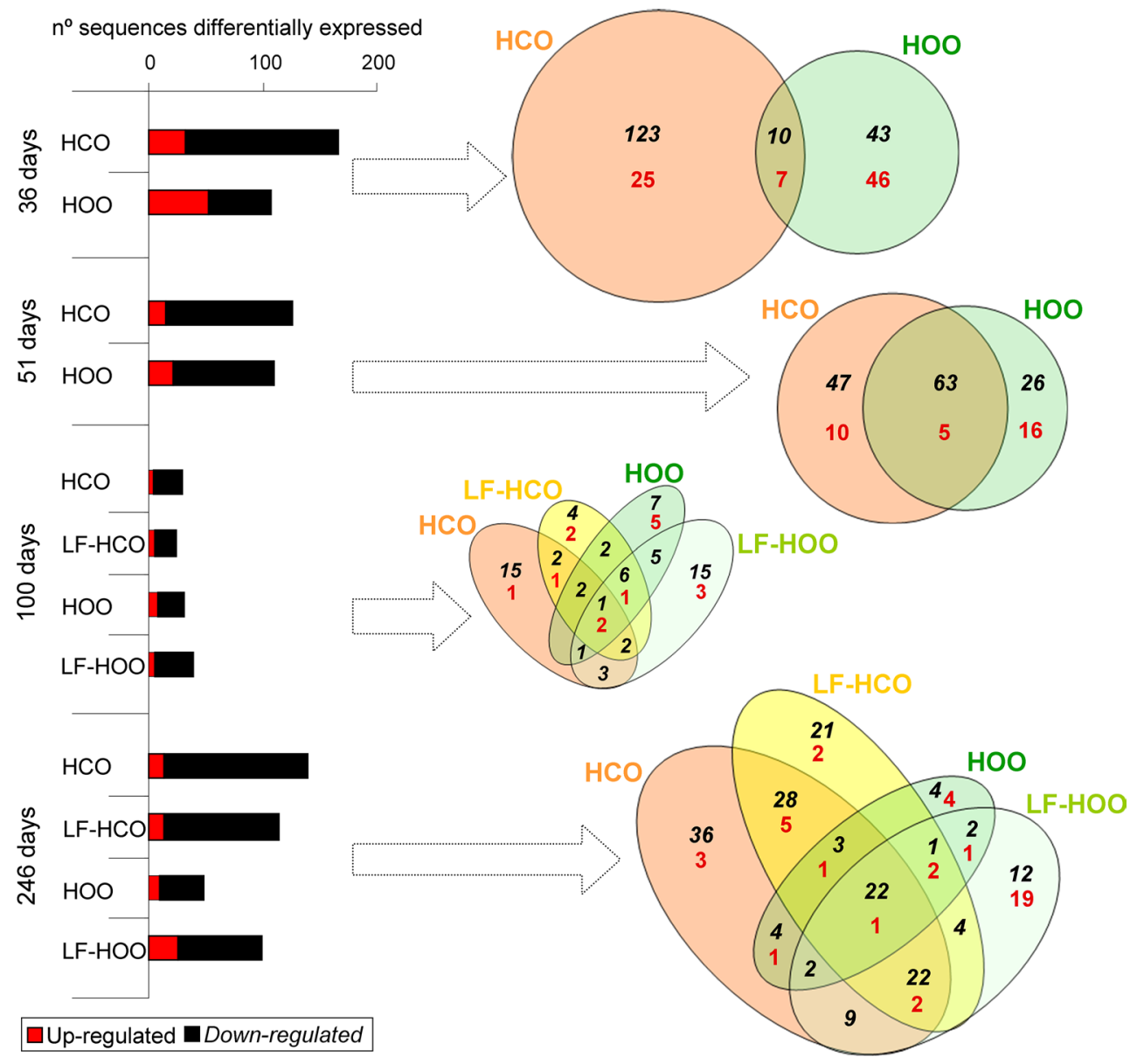

\section{Effects of high-fat diets on gene the expression profile of the mammary gland}

Results of transcriptome analyses in mammary glands from high-fat groups, in comparison with the low-fat control group, showed a larger number of down-regulated genes than up-regulated ones. Total number of modulated genes was higher in tissues from high-corn-oil diet fed animals, except for 100 days, when few differences among groups were observed (Fig. 4). For each age, lists of differentially expressed sequences were compared to identify genes modulated in common (co-modulated by effect of the high-fat diets), partially in common or unique to each specific group. Venn diagrams in Fig. 4 depict number of overlapping and specific genes. At 36 days, most of the modulated genes were specific of the group. On the contrary, at 51 days, we found an important cluster of genes modulated by the effect of both high-fat diets. As already mentioned, few sequences were modulated by 100 days. At the end of the assay (246 days), one cluster of genes corresponded to down-modulated in all high-fat diet groups. Moreover, a significant number of genes were modulated by effect of the high-corn-oil diet (in both HCO and LF-HCO groups, or specifically in each one) but not by the high EVOO diet.

Functional clustering of differentially expressed genes resulted in significantly enriched Gene Ontology (GO) categories corresponding to biological processes. Enriched categories for all genes found in each group (specific or common) are detailed in Supplementary Table 2. Among the down-modulated genes, some biological processes were generally found in several groups and at different ages, such as metabolism and response to stimulus. Thirtysix days was the age with more dissimilarities among groups, when genes down-regulated in HCO group were related to immune response, cell death, proliferation and adhesion. Cell functions such as proliferation and cell death were also overrepresented in down-modulated genes at different ages. Functional analysis was also performed dividing commonly modulated genes and specific for each group. Extended enriched categories are detailed in Supplementary Table 3 (36 days of age), Supplementary Table 4 (51 days of age) and Supplementary Table 5 (246 days of age). No significant enriched categories were found at 100 days when modulated genes were divided in specific and common. Results revealed different trends in groups 
fed the high-corn-oil diet and in the ones fed the high olive oil diet. Common genes and genes specifically modulated in the high EVOO groups were mainly related to metabolic processes. On the other hand, genes down-modulated by effect of the high-corn-oil diet had a role on apoptosis and immune system, mainly at 36 days of age.

We further selected for validation several genes related to overrepresented processes at different ages. Results of gene expression analyzed by real-time PCR are shown in Fig. 5. Genes related to lipid synthesis pathways (acetylCoA carboxylase alpha-Acaca, ATP citrate lyase-Acly, insulin-induced gene 1-Insig1) were significantly downmodulated by the effect of both high-fat diet at all ages tested. mRNA expression of genes with a role in mammary gland morphogenesis was also modulated at different ages. Thus, $\beta$-casein ( $\beta$-cas) was in general up-modulated in $\mathrm{HCO}$ group, and progesterone receptor (PgR) was upmodulated in $\mathrm{HOO}$ group at 36 days of age. Lepin (Lep) tended to be up-modulated at 36 days in HCO, and adiponectin (AdipoQ) was down-modulated in the same group at 51 days. Due to the reported importance of the ratio Lep/ AdipoQ [27], analyses of these genes were extended to all ages (Fig. 6a). Such ratio was significantly increased in $\mathrm{HCO}$ at 36 and 51 days of ages. On the other hand, differences in the expression of genes related to immune function $(\mathrm{Cd} 28, \mathrm{Cd} 3 \mathrm{~d}, \mathrm{Cd} 19)$ by high-fat diets were not validated. Moreover, expression of genes with a role in cell death (Tp53inp1 and Cebpa) showed different trends in $\mathrm{HCO}$ and $\mathrm{HOO}$ at 51 days, but results did not reach statistical significance. Finally, the mRNA levels of genes with a role in response to stimulus (Tgf $\beta 3$, S100a6, Hsp90ab1) also showed different trends in $\mathrm{HCO}$ and $\mathrm{HOO}$ groups at 100 and 246 days, specially lower levels of S100a6 in LFHOO (100 days) and lower levels of Hsp90ab1 in HCO (246 days).

\section{Effects of high-fat diets on apoptosis and $T$ cell infiltration}

In order to further investigate the effects of the high-fat diets on the expression of genes with a role in functions that may modify breast cancer susceptibility, immunohistochemical analyses of paraffin-embedded tissue were performed. As already mentioned, real-time PCR analysis in relation to genes with a role in apoptosis showed different trends in $\mathrm{HCO}$ and $\mathrm{HOO}$ groups, but results did not reach statistical significance. Detection of cell death by TUNEL showed a lower percentage of apoptotic cells in the mammary glands of animals fed the high-corn-oil diet at 36 and 51 days and tended to be lower in the glands of the animals fed the high EVOO diet at 36 days. Such effect was observed in different epithelial structures: terminal end buds (TEB), ducts, lobules and considering all of them
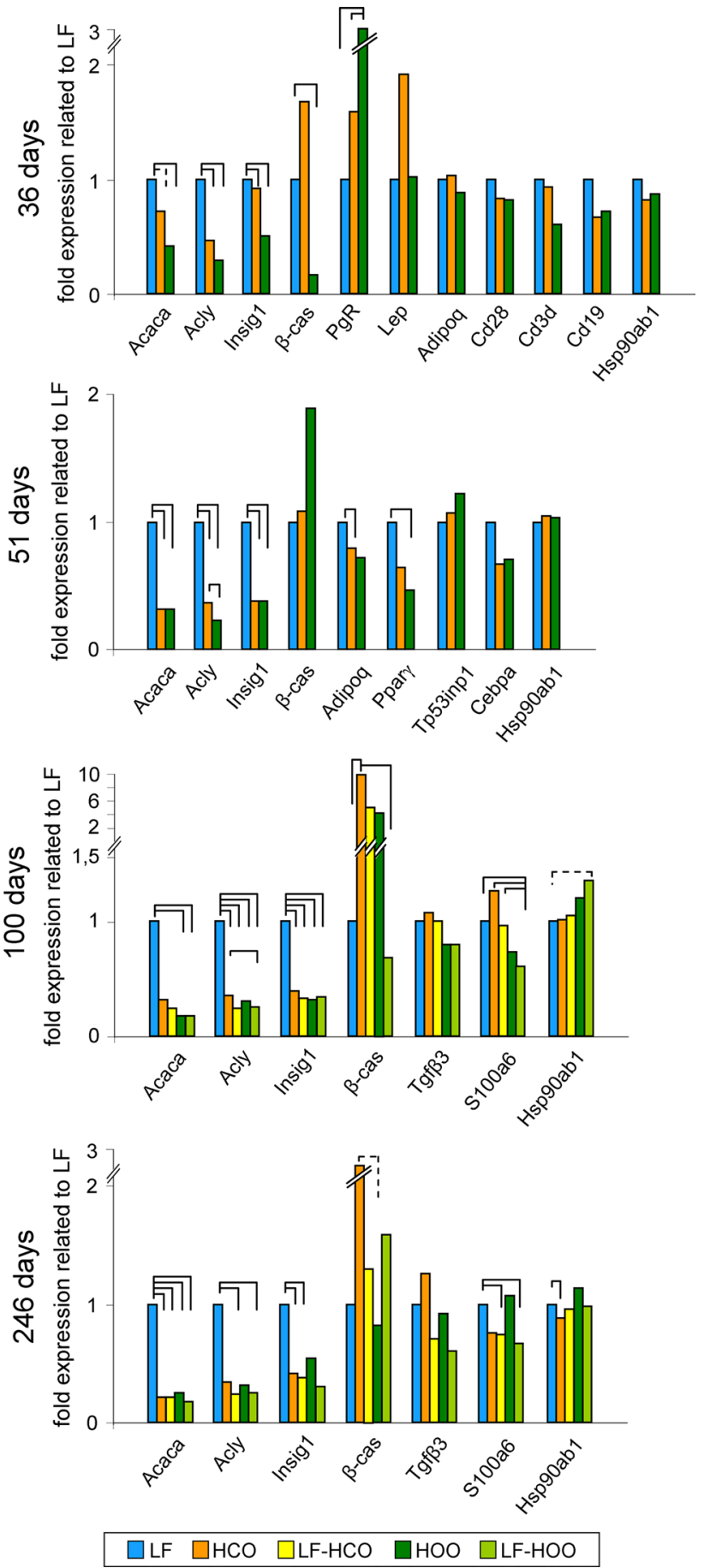

Fig. 5 Real-time PCR validation of genes found as modulated in the microarrays analysis. Data represent relative gene expression (median) (36 and 51 days $N=6 /$ group; 100 days $N=5 /$ group, 246 days $N=20$ /group). Full lines connecting groups indicate statistically significant differences $(p<0.05)$, and dotted lines indicate differences close to significance $(p<0.1)$, Mann-Whitney's $U$ test

(Fig. 6b). These effects of the diets were observed at peripubertal ages, but not in adulthood (results not shown). Moreover, we assessed by immunohistochemistry the 
a
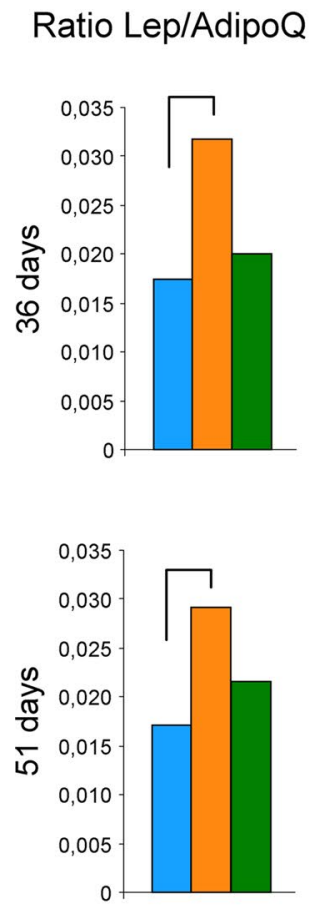

b

Apoptotic cells
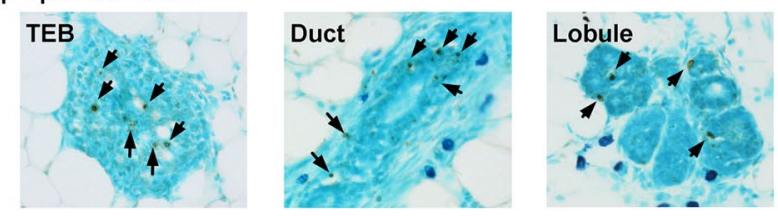

TEB
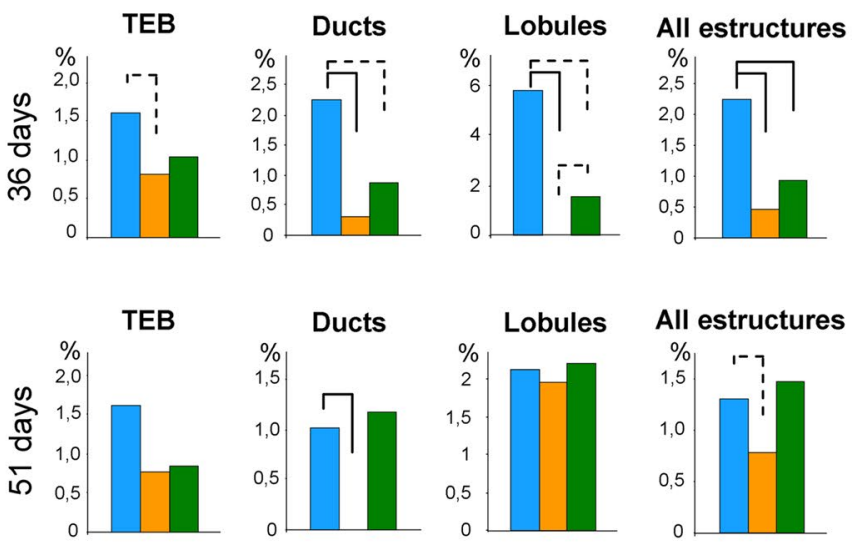

$\square$ LF $\square \mathrm{HCO} \square \mathrm{HOO}$
C
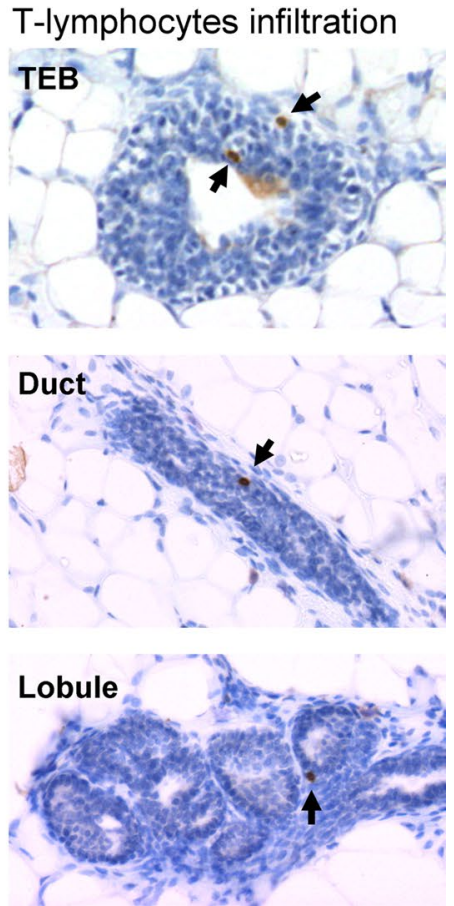

Fig. 6 Ratio between leptin and adiponectin expression (Lep/AdipoQ) in mammary glands of the experimental groups at peri-pubertal ages ( $N=6$ /group) (a). Detection of apoptosis in mammary epithelial structures and percentage of apoptotic cells at 36 and 51 days in terminal end buds (TEB), ducts, lobules, as well as considering all structures $(N=8-21$ each structure/group/age); arrows indicate posi- tive cells (b). Detection of T cells by immunohistochemistry in TEB, ducts and lobules; arrows indicate positive cells (c). Full lines connecting groups indicate statistically significant differences $(p<0.05)$, and dotted lines indicate differences close to significance $(p<0.1)$, Mann-Whitney's $U$ test

age, tumor-bearing animals and total tumor yield were increased in both high-corn-oil diet groups (HCO and LF$\mathrm{HCO}$ ), in relation to LF-HOO and control groups. Groups fed the high-corn-oil diet had also the highest value of total tumor volume, but no statistical analysis can be performed since this is a quantitative parameter with only one value per group. At the end of the assay, all the carcinogenesis parameters were increased in all groups (Fig. 7c). Groups of animals fed the corn oil-enriched diet showed the highest percentage of tumor-bearing animals, significantly different in comparison with control and HOO groups. Total number of tumors was increased in $\mathrm{HCO}$ (significantly higher values than control and HOO), followed by LF-HCO and LFHOO (increased compared to control). Finally, LF-HCO had also at that age the highest value of total tumor volume (Fig. 7c).

\section{Discussion}

We have previously reported that high-fat intake influence DMBA-induced breast cancer susceptibility [3, 4, 28, 29]. In this work, we have investigated whether diets rich in 
Fig. 7 Epithelial proliferative lesions in whole-mounted mammary glands: hyperplasia $(\mathrm{H})$, small intra-ductal proliferation (IDP1), large intra-ductal proliferation (IDP2), tumor (T); scale bar $250 \mu \mathrm{m}$ (a). Carcinogenesis parameters at 105 days of age (52 days after DMBA induction, $N=20$ /group) (b). Carcinogenesis parameters at the end of the assay (236-256 days of age, $N=20 /$ group) (c). Full lines connecting groups indicate statistically significant differences $(p<0.05)$; dotted lines indicate differences close to significance $(p<0.1)$, Mann-Whitney's $U$ test a
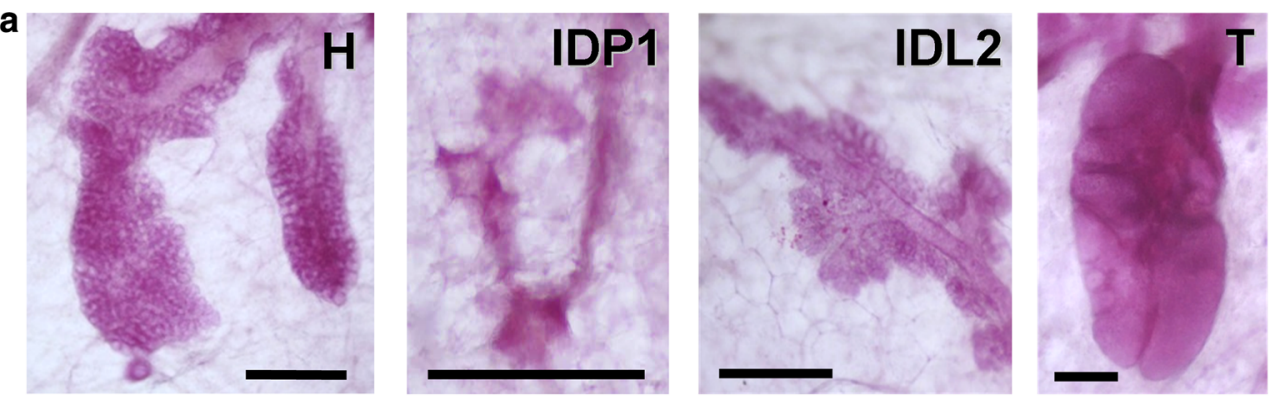

b Tumor-bearing animals

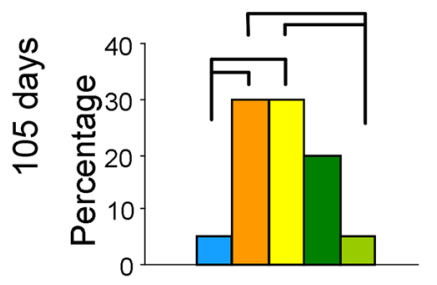

C

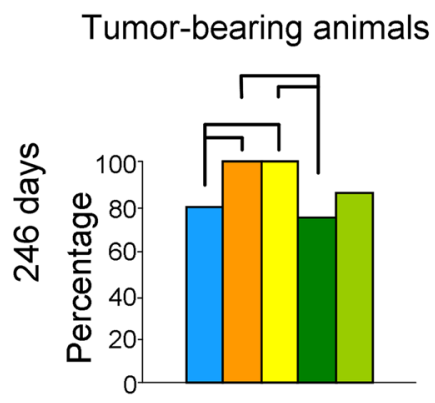

Tumor yield

Total tumor volume

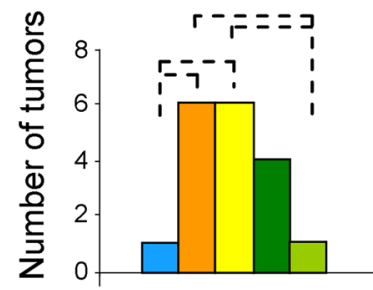

Tumor yield

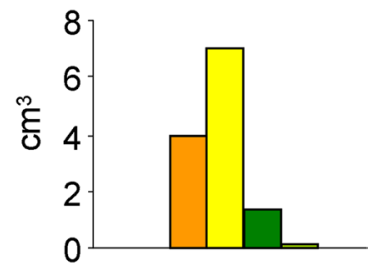

Total tumor volume
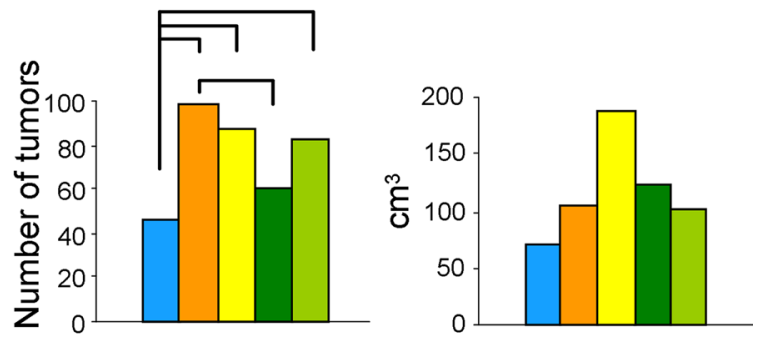

\section{LF $\square$ HCO $\square$ LF-HCO $\square$ HOO $\square$ LF-HOO}

corn oil or in extra-virgin olive oil have a different influence on experimental mammary carcinogenesis through a differential effect on development (including body growth and metabolism), also affecting the molecular context of the mammary gland. The high-corn-oil diet, when administered from weaning, increased body weight and mass, but the high EVOO diet did not, probably related to an increase in energy expenditure through mitochondrial uncoupling proteins. These diets have different effects on mammary gland transcriptome profile, especially short after dietary intervention, with the high-corn-oil diet down-regulating genes related to immune system and apoptosis. Further immunohistochemical investigation suggested a decrease in apoptosis in the mammary epithelial structures of animals fed the high-corn-oil diet, what can be one of the mechanisms of its clear stimulating influence on mammary carcinogenesis.

Nutritional factors influencing growth, maturation and adiposity may have an impact on the susceptibility to mammary transformation [2]. Actually, obesity and early menarche are well-known risk factors for human breast cancer [2, 12]. Here we have observed a different influence of the high-corn-oil and EVOO diets on body weight when administered from weaning, although no changes were evident if diets were administered after puberty (when the carcinogenesis has been already initiated). Hence, the $\mathrm{HCO}$ group, but not the HOO one, had higher body weight throughout the study and body mass at the end of the assay. Considering that both high-fat diets have an excess in lipid content, these data highlight that not only the caloric intake but also the type of lipid consumed are important factors in the control of body weight. The results obtained suggest that these two types of lipid may have different effects on energy intake/expenditure balance, and are also in accordance with other authors reporting that olive oil produce less body-weight gain than saturated fat [30] and corn oil [31]. Thus, we further analyzed whether these diets have an effect on the hepatic expression of genes with a role in energy balance, lipid transport and metabolism, such as PPAR $\alpha$ and its target genes CPT1a, L-FABP and UCP2. A different effect was only observed in UCP2, up-regulated 
mainly by effect of the high EVOO diet. In accordance with this, an olive oil diet has also been reported to increase the expression of UCPs in brown adipose tissue and muscle $[31,32]$. UCPs are mitochondrial proteins uncoupling oxidative phosphorylation and therefore dissipating energy as heat. All together, these results suggest an increase in liver lipid uptake and $\beta$-oxidation by effect of both high-fat diets [33], probably related to the physiological processing of lipids, but an increase in energy dissipation (thus decreasing the ratio energy intake/expenditure) only by effect of the high EVOO diet. Although caution must be applied when comparing human data and animal models, these results are in accordance with epidemiological data showing that Mediterranean diet, with the main source of fat being olive oil, has a protective effect on body weigh gain and obesity [10, 11, 34].

Changes in body growth, sexual maturation and energy intake/expenditure balance may influence breast cancer susceptibility [2, 12]. Aiming to investigate molecular mechanisms driving the effects of the experimental diets, we determined the gene expression profile of the mammary gland at 36, 51, 100 and 246 days of age. These ages are of special interest to study the susceptibility to malignant transformation and the promotion of cancer. At 36 days, just after puberty onset, the mammary gland is actively developing, period which extends to 51 days. Moreover, this time point (51 days) is of particular relevance as it is within the window of maximum susceptibility to malignant transformation [35], 2 days before the induction with DMBA. After induction, around 100 days of age (47 days post-DMBA) carcinogenesis begins to clinically manifest in all groups. With the DMBA dose used ( $5 \mathrm{mg}$ ), in order to study the promotion of cancer, the assays are typically extended to 200-250 days.

Transcriptomic analysis in mammary glands showed different effects of corn oil and extra-virgin olive oil. In general, the high-n-6 PUFA diet regulated a larger number of sequences. By 36 days of age, the diets induced a clear specific gene expression signature, and few genes were commonly modulated by both diets. The total number of modulated sequences decreased thereafter and an important set of genes were commonly modulated (co-regulated by the high-fat diets). Functional clustering of modulated genes resulted in significantly enriched categories showing different trends also depending on the diet. At different ages tested, co-regulated genes and genes modulated only in EVOO groups were frequently related to metabolism. Genes only down-modulated by the corn oil-based diet were recurrently involved in immune system and apoptosis, functions related to cancer susceptibility [36, 37]. At the light of those results, the effects of high-fat diets seem to be stronger and more specific after a short period (at 36 days, 12 days after beginning of dietary intervention) than after longer administration, and both diets induce different changes in the mammary gland. In rat colonic mucosa, it has also been described specific gene expression signatures depending on the type of lipid intake (fish oil, corn oil or olive oil [38]).

Validation analysis by real-time PCR confirmed the comodulation of genes with a role in lipid metabolism, specially the inhibition of lipid synthesis, as a response to the high lipid intake that extends to the adaptation to chronic consumption. Both high-fat diets clearly down-regulated the rate-limiting enzyme in fatty acid synthesis (Acaca), the enzyme for the synthesis of cytosolic acetyl-CoA (Acly) and the key enzyme that regulates cholesterol concentrations (Insig1). We also observed changes in $\beta$-casein and progesterone receptor at puberty that were concordant with the advanced puberty reported [19], and an increase in $\beta$-casein in adulthood compatible with the higher degree in gland density in the HCO group previously observed [19]. Regarding the adipocytokines, leptin and adiponectin, they have been reported to exert antagonistic effects on mammary gland development $[27,39]$. Thus, we calculated the ratio leptin/adiponectin finding a significant increase by effect of the corn oil-enriched diet at peri-pubertal ages, what is compatible with a more proliferative gland [27] just before DMBA induction. On the other hand, results for immune system genes showed a great variability among samples, and differences found in the transcriptomic analysis were not validated. Since most of the immune system genes found in the microarrays were lymphocyte $\mathrm{T}$ markers, immunohistochemical detection of $\mathrm{T}$ cells was carried out, observing few cells in the different epithelial structures. Intra-epithelial $\mathrm{T}$ cells have been identified in the normal human breast, but little is known about their specific function [40]. As there is hormonal control of the immune system that may contribute to mammary development via recruitment of immune cells [40], the lack of evident changes in immune system genes may be related to sample collection at different stages of the estrus cycle.

An important cluster of genes differentially regulated by the high-fat diets was the one related to apoptosis. Thus, by 51 days of age, results suggested a decrease in apoptosis genes such as CCAAT/enhancer binding protein alpha (Cebpa) and tumor protein p53 inducible nuclear protein 1 (Tp53inp1) by effect of the n-6 PUFA-enriched diet. Cebpa is regulated by adiponectin, and both were down-regulated in the mammary glands of HCO group. Both Cebpa and Tp53inp1 can be down-regulated in breast cancer [41, 42], and although validation also suggested a different effect of those diets in their expression, evident differences were not obtained. However, further histological detection of apoptotic cells by TUNEL confirmed a decrease in apoptosis in the epithelial cells in HCO group. These data suggested that changes in the apoptotic capacity of the mammary gland may be one of the molecular mechanisms by which 
the corn oil-enriched diet has a clear stimulating effect on mammary carcinogenesis.

In the DMBA-induced adult mammary gland, other genes also showed different trends depending on the diet administered, such as Tgf $\beta 3$ and Hsp90ab1, but differences did not reach statistical significance. Finally, glands from LF-HOO group had down-regulated expression of S100a6 at both adult ages tested. S100 genes have a role in breast cancer progression, and it has been reported a decrease in S100a6 concomitantly with a reduction in oxidative stress [43]. These data are in accordance with lower cancer-promoting microenvironment in the glands of rats fed the olive oil-enriched diet and can be related to the different carcinogenesis evolution observed among groups. In this sense, in mice, a high-fat diet also increased mammary cancer susceptibility and elevated the inflammatory gland environment [44].

The experimental diets have also exerted different effects on the clinical evolution of the cancer disease. In accordance with previous results [3, 4, 28, 29], the diet rich in n-6 PUFA had a clear stimulating effect on breast carcinogenesis. At day 52 after DMBA induction (105 days of age), the carcinogenesis yield was low in all groups. The study of microscopic proliferative structures showed more epithelial lesions (mainly microtumors) in groups fed the diet rich in n-6 PUFA. Clinical parameters of the carcinogenesis had also higher values in such groups, while results from the olive oil fed groups had no significant differences compared to controls. At 246 days, the carcinogenesis yield was higher in all groups and values were more similar among them, which suggests that in the long term, the progression of cancer disease ends up being stronger than the action of environmental factors. Nevertheless, there was also a clear stimulating effect of the diet rich in n-6 PUFA, whereas the groups fed the high EVOO diet were more similar to the low-fat control. There is human and experimental evidence that total intake of fat positively correlates with breast cancer risk [2, 45]. Hence, while the stimulating effect of the high-corn-oil diet is clear and strong, the more variable and weaker influence of the high EVOO diet, despite being high fat, suggests that this type of oil has some beneficial effect that may partially counteract the total fat intake. Several active components of olive oil have been suggested to have health benefits, including the MUFA oleic acid or the phenolic minor compounds [46, 47]. We previously reported in experimental mammary tumors that these two types of diet exert a different modulation of cell-signaling pathways that modifies the cell proliferation-apoptosis balance, showing higher proliferation by effect of the high corn oil and higher apoptosis by the high EVOO [48]. Results obtained in this work also suggest a different influence of these diets on breast cancer susceptibility leading a different proliferation-apoptosis balance in the pubertal mammary gland as well and inducing a different promoting microenvironment.

The importance of the high-fat intake before puberty on breast cancer susceptibility has also been reported by other authors. Prepubertal exposure to a low-n-3 PUFA diet in rat reduced cell proliferation, increased apoptosis and lowered breast cancer risk in adulthood, whereas a prepubertal highn-3 PUFA intake had opposite effects [49]. In BALB/c mice, a commercial diet rich in high fat (from corn oil and lard) induced increased mammary epithelial cell proliferation, macrophage infiltration, angiogenesis and inflammatory factors [44]. Here we have observed a different effect of diets enriched in corn oil or EVOO on peri-pubertal mammary gland compatible with an increase in susceptibility by effect of high-corn-oil intake during puberty. However, this type of oil has shown a strong promoting effect eliciting that, even if the susceptibility to malignant transformation is increased, the fast growth and progression of carcinogenesis in the animals fed that diet made no evident clinical difference upon the beginning of dietary intervention (childhood or adulthood, e.g., group HCO or LFHOO). The effect of the high EVOO diet on susceptibility seemed to be weaker, and carcinogenesis from animals fed this diet progressed more slowly (in the long term) if dietary exposure began in childhood than in adulthood. Taken together, all those results highlight the complex and sometimes subtle influence that dietary factors may have on initiation, promotion and progression of breast tumors, likely inducing diverse molecular contexts (in the tumor and in the adjacent mammary gland) that differentially influence each carcinogenesis stage.

\section{Conclusions}

The data obtained in this work suggest that the high-cornoil and EVOO diets, when administered from an early age, elicit different growth and long-term adiposity, with the high-corn-oil diet increasing body mass index and the olive oil-enriched one avoiding, at least in part, lipid storage thought modulation of mitochondrial uncoupling proteins. The dietary exposure also induced specific gene expression profile in the mammary glands, suggesting a more disrupted signature by effect of the high corn oil than by the EVOO diet. The effect of the one high in corn oil is compatible with an increase in proliferation/apoptosis balance in the mammary gland, which could be one of the mechanisms of the increased susceptibility to malignant transformation and the promoting microenvironment in this tissue. These data would be in accordance with the morphological transformation and the clinical manifestation of the carcinogenesis, clearly stimulated by the diet rich in PUFA $n-6$, and with a 
weaker effect of the EVOO diet. Taken together, the results point out the importance of dietetic habits, especially (but not exclusively) from early ages, on future breast cancer risk.

Acknowledgments The authors wish to thank Dr. Irmgard Costa for their collaboration in these studies. This work was supported by Grants from "Plan Nacional de I + D + I 2004-2007 \& 2008-2011" (AGL2006-07691/ALI \& AGL2011-24778), "Fundación Patrimonio Comunal Olivarero" (FPCO2008-165.396; FPCO2013-CF611.084), "Agencia para el Aceite de Oliva del Ministerio de Agricultura, Alimentación y Medio Ambiente" (AAO2008-165.471), "Organización Interprofesional del Aceite de Oliva Español" (OIP2009-165.646), "Departament de Salut i d'Agricultura, Alimentació i Acció Rural de la Generalitat de Catalunya" (GC2010-165.000). The sponsors had no role in the study design, data collection and analysis and interpretation of the results of this study.

Conflict of interest The authors declare that they have no conflict of interest.

Ethical standards The manuscript does not contain clinical studies or patient data.

Open Access This article is distributed under the terms of the Creative Commons Attribution 4.0 International License (http://creativecommons.org/licenses/by/4.0/), which permits unrestricted use, distribution, and reproduction in any medium, provided you give appropriate credit to the original author(s) and the source, provide a link to the Creative Commons license, and indicate if changes were made.

\section{References}

1. Ferlay J, Shin HR, Bray F, Forman D, Mathers C, Parkin D (2010) Estimates of worldwide burden of cancer in 2008: GLOBOCAN 2008. Int J Cancer 127:2893-2917

2. World Cancer Research Fund/American Institute for Cancer Research (2007) Food, nutrition, physical activity, and the prevention of cancer: a global perspective. American Institute for Cancer Research, Washington DC

3. Escrich E, Solanas M, Moral R (2006) Olive oil, and other dietary lipids, in cancer: experimental approaches. In: Quiles JL, Ramírez-Tortosa MC, Yaqoob P (eds) Olive Oil and Health. CABI Publishing, Oxford, pp 317-374

4. Escrich E, Solanas M, Moral R, Escrich R (2011) Modulatory effects and molecular mechanisms of olive oil and other dietary lipids in breast cancer. Curr Pharm Des 17:813-830

5. Prentice RL (1996) Measurement error and results from analytic epidemiology: dietary fat and breast cancer. J Natl Cancer Inst 88:1738-1747

6. Sieri S, Krogh V, Ferrari P et al (2008) Dietary fat and breast cancer risk in the European prospective investigation into cancer and nutrition. Am J Clin Nutr 88:1304-1312

7. Schulz M, Hoffmann K, Weikert C, Nöthlings U, Schulze MB, Boeing H (2008) Identification of a dietary pattern characterized by high-fat food choices associated with increased risk of breast cancer: the European Prospective Investigation into Cancer and Nutrition (EPIC)-Potsdam Study. Br J Nutr 100:942-946

8. Trichopoulou A, Bamia C, Lagiou P, Trichopoulos D (2010) Conformity to traditional Mediterranean diet and breast cancer risk in the Greek EPIC (European Prospective Investigation into Cancer and Nutrition) cohort. Am J Clin Nutr 92:620-625
9. Couto E, Boffetta P, Lagiou P et al (2011) Mediterranean dietary pattern and cancer risk in the EPIC cohort. $\mathrm{Br} \mathrm{J}$ Cancer 104:1493-1499

10. Romaguera D, Norat T, Vergnaud AC et al (2010) Mediterranean dietary patterns and prospective weight change in participants of the EPIC-PANACEA project. Am J Clin Nutr 92:912-921

11. Gotsis E, Anagnostis P, Mariolis A, Vlachou A, Katsiki N, Karagiannis A (2014) Health benefits of the mediterranean diet: an update of research over the last 5 years. Angiology 66:304-318

12. Hsieh CC, Trichopoulos D, Katsouyanni D, Yuasa S (1990) Age at menarche, age at menopause, height and obesity as risk factors for breast cancer: associations and interactions in an international case-control study. Int J Cancer 46:796-800

13. De Assis S, Hilakivi-Clarke L (2006) Timing of dietary estrogenic exposures and breast cancer risk. Ann N Y Acad Sci 1089:14-35

14. Lo CY, Hsieh PH, Chen HF, Su HM (2009) A maternal highfat diet during pregnancy in rats results in a greater risk of carcinogen-induced mammary tumors in the female offspring than exposure to a high-fat diet in postnatal life. Int J Cancer 125:767-773

15. Jump DB (2004) Fatty acid regulation of gene transcription. Crit Rev Clin Lab Sci 41:41-78

16. Escrich E, Solanas M, Segura R (1994) Experimental diets for the study of lipid influence on induced mammary carcinoma in rats: I-diet definition. In Vivo 8:1099-1106

17. Escrich E, Solanas M, Ruiz de Villa MC, Ribalta T, Muntane J, Segura R (1994) Experimental diets for the study of lipid influence on induced mammary carcinoma in rats: II-suitability of the diets. In Vivo 8:1107-1112

18. Solanas M, Hurtado A, Costa I, Moral R, Menéndez JA, Colomer R, Escrich E (2002) Effects of a high olive oil diet on the clinical behavior and histopathological features of rat DMBA-induced mammary tumors compared with a high corn oil diet. Int J Oncol 21:745-753

19. Moral R, Escrich R, Solanas M, Vela E, Costa I, de Villa MC, Escrich E (2011) Diets high in corn oil or extra-virgin olive oil provided from weaning advance sexual maturation and differentially modify susceptibility to mammary carcinogenesis in female rats. Nutr Cancer 63:410-420

20. Bernardis LL (1970) Prediction of carcass fat, water and lean body mass from Lee's "nutritive ratio" in rats with hypothalamic obesity. Experientia 26:789-790

21. Costa I, Solanas M, Escrich E (2002) Histopathologic characterization of mammary neoplastic lesions induced with 7,12 dimethylbenz(alpha)anthracene in the rat: a comparative analysis with human breast tumors. Arch Pathol Lab Med 126:915-927

22. Irizarry RA, Hobbs B, Collin F, Beazer-Barclay YD, Antonellis KJ, Scherf U, Speed TP (2003) Exploration, normalization, and summaries of high density oligonucleotide array probe level data. Biostatistics 4:249-264

23. Gentleman R, Carey V, Huber W, Irizarry R, Dudoit S (2005) Bioinformatics and computational biology solutions using $\mathrm{R}$ and bioconductor. Springer, New York

24. Smyth GK (2004) Linear models and empirical Bayes methods for assessing differential expression in microarray experiments. Stat Appl Genet Mol Biol 3:article 3

25. Benjamini Y, Hochberg Y (1995) Controlling the false discovery rate: a practical and powerful approach to multiple testing. J R Stat Soc B 57:289-300

26. Russo J, Russo IH (1978) DNA labeling index and structure of the rat mammary gland as determinants of its susceptibility to carcinogenesis. J Natl Cancer Inst 61:1451-1459

27. Grossmann ME, Ray A, Dogan S, Mizuno NK, Cleary MP (2008) Balance of adiponectin and leptin modulates breast cancer cell growth. Cell Res 18:1154-1156 
28. Costa I, Moral R, Solanas M, Escrich E (2004) High-fat corn oil diet promotes the development of high histologic grade rat DMBA-induced mammary adenocarcinomas, while high olive oil diet does not. Breast Cancer Res Treat 86:225-235

29. Escrich E, Solanas M, Moral R (2014) Olive oil and other dietary lipids in breast cancer. In: Zappia V, Panico S, Russo GL, Budillon A, Regione FD (eds) Advances in nutrition and cancer. Springer, Berlin

30. de Wit N, Derrien M, Bosch-Vermeulen H et al (2012) Saturated fat stimulates obesity and hepatic steatosis and affects gut microbiota composition by an enhanced overflow of dietary fat to the distal intestine. Am J Physiol Gastrointest Liver Physiol 303:G589-G599

31. Oi-Kano Y, Kawada T, Watanabe T, Koyama F, Watanabe K, Senbongi R, Iwai K (2007) Extra virgin olive oil increases uncoupling protein 1 content in brown adipose tissue and enhances noradrenaline and adrenaline secretions in rats. J Nutr Biochem 18:685-692

32. Rodríguez VM, Portillo MP, Picó C, Macarulla MT, Palou A (2002) Olive oil feeding up-regulates uncoupling protein genes in rat brown adipose tissue and skeletal muscle. Am J Clin Nutr 75:213-220

33. Vial G, Dubouchaud H, Couturier K, Cottet-Rousselle C, Taleux N, Athias A, Galinier A, Casteilla L, Leverve XM (2011) Effects of a high-fat diet on energy metabolism and ROS production in rat liver. J Hepatol 54:348-356

34. Martínez-González MA, García-Arellano A, Toledo E, SalasSalvadó J, Buil-Cosiales P, Corella D, Covas MI, Schröder H, Arós F, Gómez-Gracia E et al (2012) A 14-item mediterranean diet assessment tool and obesity indexes among high-risk subjects: the PREDIMED trial. PLoS ONE 7:e43134

35. Russo IH, Russo J (1996) Mammary gland neoplasia in longterm rodent studies. Environ Health Perspect 104:938-967

36. Coussens LM, Pollard JW (2011) Leukocytes in mammary development and cancer. Cold Spring Harb Perspect Biol 3:a003285

37. Kumar R, Vadlamudi RK, Adam L (2000) Apoptosis in mammary gland and cancer. Endocr Relat Cancer 7:257-269

38. Kachroo P, Ivanov I, Davidson LA, Chowdhary BP, Lupton JR, Chapkin RS (2011) Classification of diet-modulated gene signatures at the colon cancer initiation and progression stages. Dig Dis Sci 56:2595-2604
39. Cleary MP, Ray A, Rogozina OP, Dogan S, Grossmann ME (2009) Targeting the adiponectin:leptin ratio for postmenopausal breast cancer prevention. Front Biosci (Schol Ed) 1:329-357

40. Need EF, Atashgaran V, Ingman WV, Dasari P (2014) Hormonal regulation of the immune microenvironment in the mammary gland. J Mammary Gland Biol Neoplasia 19:229-239

41. Gery S, Tanosaki S, Bose S, Bose N, Vadgama J, Koeffler HP (2005) Down-regulation and growth inhibitory role of C/EBPalpha in breast cancer. Clin Cancer Res 11:3184-3190

42. Tomasini R, Samir AA, Carrier A et al (2003) TP53INP1s and homeodomain-interacting protein kinase-2 (HIPK2) are partners in regulating p53 activity. J Bio Chem 278:37722-37729

43. Uchiyama Y, Suzuki T, Mochizuki K, Goda T (2013) Dietary supplementation with a low dose of (-)-epigallocatechin-3-gallate reduces pro-inflammatory responses in peripheral leukocytes of non-obese type 2 diabetic GK rats. J Nutr Sci Vitaminol (Tokyo) 59:541-547

44. Zhao Y, Tan YS, Aupperlee MD, Langohr IM, Kirk EL, Troester MA, Schwartz RC, Haslam SZ (2013) Pubertal high fat diet: effects on mammary cancer development. Breast Cancer Res 15:R100

45. Guthrie N, Carroll KK (1999) Specific versus non-specific effects of dietary fat on carcinogenesis. Prog Lipid Res 38:261-271

46. Hashim YZ, Eng M, Gill CI, McGlynn H, Rowland IR (2005) Components of olive oil and chemoprevention of colorectal cancer. Nutr Rev 63:374-386

47. Owen RW, Giacosa A, Hull WE, Haubner R, Würtele G, Spiegelhalder B, Bartsch H (2000) Olive-oil consumption and health: the possible role of antioxidants. Lancet Oncol 1:107-112

48. Solanas M, Grau L, Moral R, Vela E, Escrich R, Escrih E (2010) Dietary olive oil and corn oil differentially affect experimental breast cancer through distinct modulation of the p21Ras signaling and the proliferation-apoptosis balance. Carcinogenesis 31:871-879

49. Olivo-Marston SE, Zhu Y, Lee RY, Cabanes A, Khan G, Zwart A, Wang Y, Clarke R, Hilakivi-Clarke L (2008) Gene signaling pathways mediating the opposite effects of prepubertal low-fat and high-fat n-3 polyunsaturated fatty acid diets on mammary cancer risk. Cancer Prev Res (Phila.) 1:532-545 